\title{
SOFT PRODUCTION OF PHY MESON IN PHOTON PROTON INTERACTION
}

\author{
Khalid M. A. Hamad ${ }^{1}$, Husam Mohamed Saddaa Hamad \\ Department of Physics, College of Science, Al-Anbar University, Iraq \\ 1 khalid_hamad2002@yahoo.com
}

\begin{abstract}
In the present model the photoproduction of the $\varnothing$ meson cross section is calculated. The contribution from soft pomeron exchange is calculated using DL model. It is found that this model is over-estimated the data. A mass correction factor related to the masses of $\rho$ and $\emptyset$ mesons is used to normalize the DL model. The hard pomeron contribution is also calculated using The pQCD model. A reasonable agreement between the model and the data is obtained..
\end{abstract}

Keywords: Soft Production, phy Meson Production.

\section{INTRODUCTION}

Diffraction is a familiar phenomenon in particle physics. The hadron -hadron scattering is described by exchange of a virtual, colorless and neutral object carrying no quantum numbers called the pomeron. Donnachie and Landshoff (DL) have performed a simple Regge parameterization of the total cross section. The parameterization is a sum of two terms, the pomeron term and the reggeon term[1]

$\sigma_{T}=A s^{\epsilon}+B s^{-\eta}$

With $\mathrm{s}$ is the squared of the total energy and $\mathrm{t}$ is the transfer momentum. The power $\epsilon$ is related to the pomeron trajectory

$\alpha_{p}(t)=\alpha_{p}(0)+\alpha_{p}^{\prime} \mathrm{t}$

with $\alpha_{p}(0)=1+\epsilon$ is the pomeron intercept. Similarly $\eta$ is related to the Regge trajectory

$\alpha_{R}(t)=\alpha_{R}(0)+\alpha_{R}^{\prime} t$

with $\alpha_{R}(0)=1-\eta$ is the Reggeon intersect. Fit for hadronic cross sections shows that the powers in (1) are universal with $\epsilon=0.0808$ and $\eta=0.4525$, while the constants $\mathrm{A}$ and $\mathrm{B}$ depend on the type of the interacting particles. According to (1) the cross sections increase with $s$ at high values of $s$ due to pomeron exchange. Because the value of $\epsilon$ is small the increase of $\sigma_{T}$ with energy is gradual and soft, so the exchanged pomeron is called a soft pomeron. Soft pomerons also dominate the elastic scattering of hadrons at high $s$ and small t. The process of photon hadrons interaction[2] is also dominated by a soft pomeron exchange. According to the vector meson dominance model (VDM) $[3,4]$, real photon and virtual photon with a small mass may be resolved into vector mesons, thus the photon interacts with hadrons through vector mesons, and the cross section is then dominated by a soft pomeron exchange. Investigations of the deep inelastic scattering (DIS) show that the structure function $f_{2}\left(Q^{2}, x\right)$ behaves like $x^{-\lambda}$ at small values of $\mathrm{x}$, with $\lambda$ is a slow growing function of $Q^{2}$ (the photon mass squard)[5]. The growth is associated with the behaviour of the gluon structure function at small $\mathrm{x}$. The cross sections show a steep increase with energy at 
high $Q^{2}$. This increase is much greater than would be expected from soft pomeron exchange [6], the process is then called a hard process and the exchanged pomeron is a hard pomeron. Investigations indicate that the transition from the soft region where $Q^{2}=0$ to the hard region of high $Q^{2}$ is smooth. The production of the vector mesons in the hard region is described by the perturbative quantum chromo dynamic (pQCD). In this region one or more of the variables $Q^{2}, t$ and $m_{v}^{2}$ should be high [7], where $m_{v}$ is the vector meson mass. Therefore, at small $t$, the photo production of vector mesons is either soft or hard depending on $m_{v}$. Experimentally the photoproduction of $\rho[8,9]$ and $\omega$ [10] is soft, while the photoproduction of heavy vector mesons $(J / \psi, \Upsilon \ldots)$ is hard. These hard processes are explained in terms of pQCD [11-15], where $m_{v}^{2} / 4$ replaces $Q^{2}$ as a measure of the process hardness. To ensure a smooth transition from the soft mass region to the high mass region, the photoproduction of $\phi$ meson should involve both the soft and the hard processes.

Several authors have investigated the $\varnothing$ meson photoproduction. DL [1] and Block et. al [16] show that the total cross section of the $\varnothing$ meson photoproduction behaves like $w^{4 \epsilon_{0}}$ and the forward slope like $4 \alpha_{p}^{\prime} \ln w$. These investigations depend on a comparison between integrated cross section data $[1,11$, 16] taken by different groups who have used different but not consistent methods. Furthermore, the integrated cross sections involve a slope parameter which is shrinking with energy, so it is hard to interpret them as a fixed power of $\mathrm{w}$. To overcome these difficulties Ferreira and Maor [17] have used differential cross sections as they are reported by the experiments $[11,18-20]$. The analysis shows that a good fit for the data is given by $\frac{d \sigma}{d t}(t=0)=A w^{4 \lambda}$

Where $A=0.67 \pm 0.09$ and $\lambda=0.133 \pm 0.012$. we notice that the value of $\lambda$ is higher than the value of the soft pomeron intercept at $\epsilon=0.0808$ which indicates a hard contribution.

For a comparison, the authors used the soft pomeron power $4 \epsilon=0.3232$ and found that the best fit in this case is obtained if $\mathrm{A}=1.21 \pm 0.06$. However, the authors concluded that the quality of the fit is in the favor of the hard behavior in (4). In their model Ferreira and Maor have calculated the hard contribution to the $\varnothing$ meson photoproduction using the $\mathrm{PQCD}$. Since the amplitude is a sum of two terms, the soft and the hard amplitudes, the authors used the form $A s^{\epsilon}$ for the soft amplitude with $\mathrm{A}$ as an adjusting parameter to fit the data. In the present model, the soft contribution is calculated without any adjusting parameter. As the hard amplitude in the $\varnothing$ meson photoproduction has arisen because $m_{\phi}$ is high compared with $m_{\rho}$ where the process is soft, then the comparatively high value of $m_{\phi}$ should also affect the soft amplitude. The amplitude of soft photoproduction of $\phi$ is similar to that of $\rho$ meson [2], but the effect of the mass is included in the calculation. Our procedure is to discuss the hard production of the $\rho$ meson using our previous calculation [21] with some minor modifications.

The $\rho$ meson photoproduction is then obtained by taking $Q^{2}=0$. The result should be the same as that given by DL calculation [2]. The same procedure is applied for the $\varnothing$ production taking into account the effect of increasing the vector meson mass from the soft $m_{\rho}$ to $m_{\phi}$. Finally, the photoproduction of $\phi$ meson(soft + hard) is compared with data. 


\section{DIFFRACTIVE $\rho$ MESON PHOTOPRODUCTION}

The diffractive Photoproduction of $\rho$ meson has been calculated by DL using the vector meson Dominance and the additive quark models [2]. The forward differential cross section for $\gamma p \rightarrow \rho p$ is given by $[4,6]$

$\frac{d \sigma}{d t}(\gamma p \rightarrow \rho p ; t=0)=\frac{4 \pi \alpha}{f_{\rho}^{2}}$

$\frac{d \sigma}{d t}(\rho p \rightarrow \rho p ; t=0)$

where $\frac{4 \pi}{f_{\rho}^{2}}=0.494$ is the $\rho-$ photon coupling[6]. According to Regge model the $\rho p \rightarrow \rho p$ forward elastic amplitude is given by $[6,22]$

$A=A_{P} S^{\alpha_{P}(0)} e^{-i \frac{\pi}{2} \alpha_{P}(0)}+A_{R} S^{\alpha_{R}(0)} e^{-i \frac{\pi}{2} \alpha_{R}(0)}$

Where $A_{P}$ and $A_{R}$ are the couplings of the pomeron and Reggeon to the interacting particles. The differential cross section is then given by

$\frac{d \sigma}{d t}(\rho p \rightarrow \rho p ; t=0)=\frac{1}{16 \pi s^{2}}|A|^{2}$

According to the additive quark model the $\rho p$ total cross section is given by the average of the $\pi^{+} p$ and $\pi^{-} p$ total cross sections, with[6]

$\sigma_{T}(\rho p)=13.6 s^{0.8080}+31.85 s^{-0.4525}$

The optical theorem relates the total cross section in(8) to the imaginary part of the amplitude in (6), then we get [6]

$A_{P}=13.74$ and $A_{R}=41.95$

\section{DIFFRACTIVE $\rho$ MESON ELECTROPRODUCTION}

It is familiar that diffractive electroproduction of vector meson is studied in terms of the $\mathrm{pQCD}$. A different approach for the process is represented by the diagram in Fig. (1b). According to the model [22], when the photon becomes off mass shell it may fluctuate into quark-anti quark system. As a result, the exchanged object couples to the photon - vector meson vertex through a quark loop as shown in $\operatorname{Fig}(1 \mathrm{~b})$. If $Q^{2}$ approaches zero the electroproduction process is reduced to the photoproduction processes in Fig.(1a).

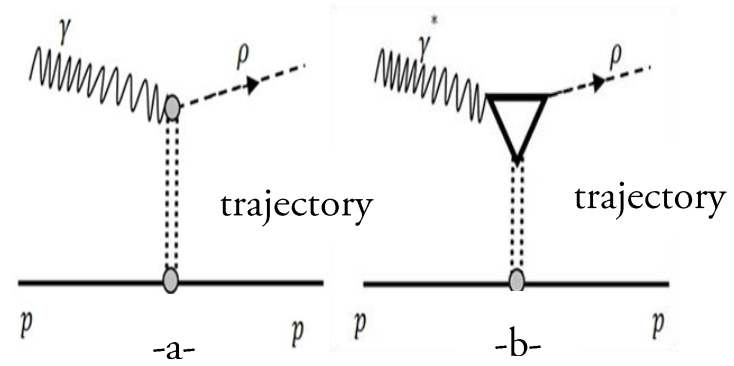

Fig. 1 Schematic representation of $\rho$ production a- photon proton interaction $b$ - virtual photon proton interaction. The triangle represents the trajectory- photon- meson coupling

At $Q^{2}=0$ the vertex should disappear and its value equals to one. Accordingly, the cross section for electroproduction of vector meson is regarded as the photoproduction of vector meson multiplied by the vertex form factor. This form factor is given by the form [21] $(4 \pi)^{2} f\left(Q^{2}, 0, m_{v}^{2}\right)=\frac{1}{Q^{2}+m_{v}^{2}}\left(\pi^{2}-\right.$ $\left.\ln ^{2}\left(\frac{1+B_{2}}{1-B_{2}}\right)+\quad \ln ^{2}\left(\frac{B_{1}+1}{B_{1}-1}\right)\right)$

Where $B_{1}=\left(1+\frac{4 m_{q}^{2}}{Q^{2}}\right)^{\frac{1}{2}}$

$$
B_{2}=\left(1-\frac{4 m_{q}^{2}}{m_{v}^{2}}\right)^{\frac{1}{2}}
$$

With $m_{q}$ is the quark mass. The cross section for the electroproduction of $\rho$ meson is then given as

$\frac{d \sigma}{d t}\left(\gamma^{*} p \rightarrow \rho p ; 0\right)=$

$$
\left(\frac{m_{\rho}^{2}}{m_{\rho}^{2}+Q^{2}} f\left(Q^{2}, 0, m_{\rho}^{2}\right)\right)^{2} \frac{d \sigma}{d t}(\gamma p \rightarrow
$$$$
\rho p ; t=0)
$$

The two sides of equation (13) are equal at $Q^{2}=0$, which requires that the equation is written as

$\frac{d \sigma}{d t}\left(\gamma^{*} p \rightarrow \rho p ; t=0\right)=$ $\left[\frac{m_{\rho}^{2}}{m_{\rho}^{2}+Q^{2}} \frac{\left(f\left(Q^{2}, 0, m_{\rho}^{2}\right)\right.}{\left(f\left(0,0, m_{\rho}^{2}\right)\right.}\right]^{2} \frac{d \sigma}{d t}(\gamma p \rightarrow \rho p ; t=0$

At $Q^{2}=0$ the form factor in (10) is given by $(4 \pi)^{2} f\left(0,0, m_{v}^{2}\right)=$

$\frac{1}{m_{v}^{2}}\left(\pi^{2}-\quad \ln ^{2}\left(\frac{1+B_{2}}{1-B_{2}}\right)\right)$

We notice that as $Q^{2} \sim 0$, the factor in the square brackets in (14) is reduced to one, then 
the extrapolated process is given by the real process.

\section{SOFT PHOTOPRODUCTION OF $\varnothing$ MESON}

For $\emptyset$ meson photoproduction, Equation (5) becomes

$\frac{d \sigma}{d t}(\gamma p \rightarrow \emptyset p ; t=0)=\frac{4 \pi \alpha}{f_{\varnothing}^{2}} \quad \frac{d \sigma}{d t}(\varnothing p \rightarrow$ $\emptyset p ; t=0)$

With the coupling constant [25] is given as $\frac{4 \pi}{f_{\varnothing}^{2}}=\frac{1}{18.4}$.According to the Okubo-Zweig rule, the reggeon contribution is suppressed, then [1]

$\sigma_{T}(\emptyset p)=10.01 w^{0.1616}$

Following the same procedure applied for $\rho$ meson in Equations $(6,7,8$, and 9) we get:

$A_{P}=10.1 \mathrm{mb}$

then

$\frac{d \sigma}{d t}(\gamma p \rightarrow \emptyset p, t=0)=$

$\frac{1}{16 \pi \times} \frac{4 \pi \alpha}{0.388 \times f_{\varnothing}^{2}}\left[A_{P} S^{0.0808}\right]^{2}$

A comparison of DL cross section in (19) with data is given in Fig. (2). we notice that the prediction from DL type in (19) is higher than the data. This is expected since VMD is an approximate model. Furthermore, the mass of the $\varnothing$ vector meson is relatively high and should be included in the calculation. For $\varnothing$ meson production (14) becomes

$\frac{d \sigma}{d t}\left(\gamma^{*} p \rightarrow \emptyset p, t=0\right)=$

$\left[\frac{\left(f\left(Q^{2}, 0, m_{\varnothing}^{2}\right)\right.}{\left(f\left(0,0, m_{\rho}^{2}\right)\right.}\right]^{2} \frac{1}{16 \pi} \frac{4 \pi \alpha}{0.388 \times f_{\varnothing}^{2}}\left[A_{P} S^{0.0808}\right]^{2}$

At $Q^{2}=0$ we get

$$
\begin{aligned}
& \frac{d \sigma}{d t}(\gamma p \rightarrow \emptyset p, t=0) \\
& =\left[\frac{\left(f\left(0,, 0, m_{\varnothing}^{2}\right)\right.}{\left(f\left(0,0, m_{\rho}^{2}\right)\right.}\right]^{2} \frac{1}{16 \pi} \frac{4 \pi \alpha}{0.388 \times f_{\varnothing}^{2}} \\
& \quad \times\left[A_{P} s^{0.0808}\right]^{2}
\end{aligned}
$$

The factor in the square bracket is a mass correction factor which takes into the account the effect of the $\emptyset$ meson mass. In general, the production of any vector meson with $m_{v}>$ $m_{\rho}$ should involve a similar mass correction factor. The prediction form the DL model in (21) is then suppressed by this factor. For $\varnothing$ meson production, this prediction is represented by the dashed curve in fig. (2).

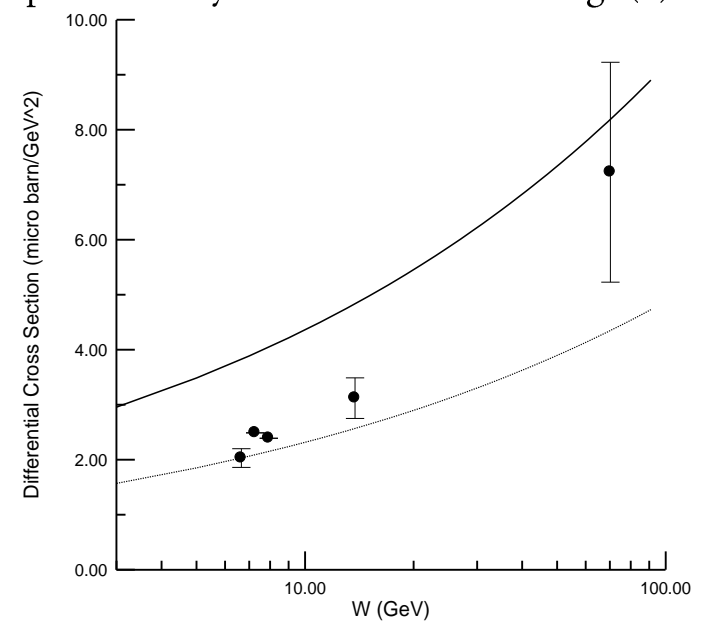

Fig.(2) $\frac{d \sigma}{d t}(\gamma p \rightarrow \emptyset p, t=0)\left(\frac{\mu b}{G e V^{2}}\right)$, the solid curve is the DL soft model, while the dashed curve is the DL soft model normalized by the mass correction factor. The data points are explained in [17] .

\section{THE HARD MODEL}

The pQCD model is also used to calculate the hard contribution to the process. Assuming non relativistic wave functions for the vector mesons we get [17]

$\frac{d \sigma}{d t}(\gamma p \rightarrow \emptyset p, t=0)=$

$\frac{\alpha_{S}^{2} \Gamma_{e e}^{\emptyset} 16 \pi^{3}}{3 \alpha m_{\varnothing}^{5}}\left[x G\left(x, \frac{m_{\varnothing}^{2}}{4}\right)\right]^{2}$

where $x=\frac{m_{\emptyset}^{2}}{s}$, with $s$ is the invariant total energy squared and $\Gamma_{e e}^{\emptyset}$ is the partial decay width of $\varnothing \rightarrow e^{+} e^{-}$

In this calculation we require the gluon structure function $x G\left(x, Q^{2}\right)$ at

$Q^{2}=\frac{m_{\varnothing}^{2}}{4}=0.26$. This value is below the threshold value $Q_{0}$. The following linear extrapolation is used in the small $Q^{2}$ to extrapolate the structure function to small $Q^{2}$,with[26]

$\mathrm{X} \mathrm{G}\left(\mathrm{x}, Q^{2}\right)=\frac{Q^{2}}{Q_{0}^{2}} \mathrm{x} \mathrm{G}\left(\mathrm{x}, Q_{0}^{2}\right)$ with $Q^{2}<Q_{0}^{2}$ 
The GRV98[27 ] program is used to extract the gluon structure function required in (22), where $Q_{0}^{2}=0.8 \mathrm{GeV}^{2}$. The strong coupling constant $\alpha_{s}$ at $Q^{2}=0.26 \mathrm{GeV}^{2}$ is calculated in the program and is given approximately by $\frac{\alpha_{S}\left(Q^{2}\right)}{4 \pi} \approx \frac{1}{\beta_{0} \ln \left(\frac{Q^{2}}{\Lambda^{2}}\right)}$

where $\beta_{0=} 9$. In the leading order $\Lambda^{2}=$ $4.1616 \times 10^{-2}$. The PQCD calculation is represented by the dashed curve in Fig.(3), while the dotted curve represents the corrected DL soft model. The total contribution is given by the solid curve. A reasonable agreement with the data is evident in the figure.

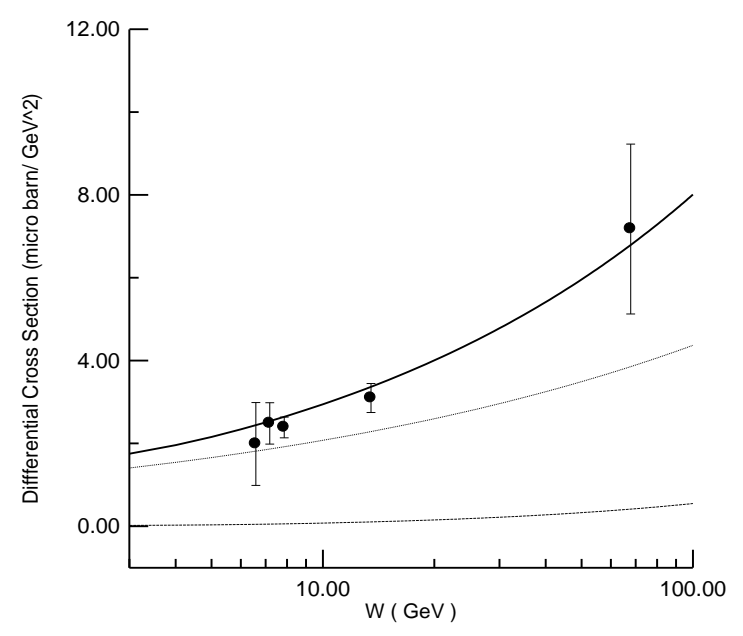

Fig.(3) $\frac{d \sigma}{d t}(\gamma p \rightarrow \emptyset p, t=0)\left(\frac{\mu b}{G e V^{2}}\right)$, the dotted curve is the DL soft model normalized by the mass correction factor, the dashed curve is the hard term using the pQCD calculation. The solid curve is the contribution from both terms. The data points are explained in[17] .

\section{CONCLUSION}

As the VDM is not applicable for vector mesons with masses higher than the Rho meson mass, a mass correction factor is included in the present work. The model is applied for phy meson production. A comparison with data shows a reasonable agreement. The model is regarded as an extension of the VMD model to the mesons with high masses.

\section{REFERENCES}

[1] A. Donnachie P. Landshoff , Total Cross Sections, Phys. Lett. B296 (1992) 227-232

[2] T. Ahmed et al.(H1 Coll.) , Total Photoproduction Cross Section Measurement at HERA Energies, Phys. Lett. B299 (1993) 374384

[3] D. Schildknecht, Vector Meson Dominance, arXiv: hep-ph/ 0511090 (2005)

[4] D. Schildknecht, Vector Meson Dominance, Photo and Electroproduction from Nucleons, Springer Tracts in Modern Physics v63 (1972) 57-97

[5] A. Capella, A. Kaidalov, C. Merino, J. Tran Than Van, Structure Functions and Low x Physics, Phys. Lett. B337, (1994) 358-366

[6] A. Donnachie P. Landshoff, Exclusive Vector Meson Production at HERA, Phys. Lett. B348 (1995) 213-218

[7] J. A. Crittenden et al., Scale Issues In HighEnergy Diffractive Vector Meson Production, hep-ex /9806020

[8] M. Derrick et al. (ZEUS Coll.), Measurement of Elastic $\rho^{0}$ Photoproduction at HERA, Z-Phys. C64, (1995)39-54

[9] S. Aid. et al.(H1 Coll.), Elastic Photoproduction of $\rho^{0}$ Mesons at HERA, Nucl. Phys. B463, (1996) 3-32

[10] M. Derrick et al. (ZEUS Coll.), Measurement of Elastic Omega Photoproduction at HERA, Z. Phys. C73 (1996) 73-84

[11] M. Derrick et al. (ZEUS Coll ), Measurement of Elastic Phi Photoproduction at HERA, Phys. Lett. B377 (1996) 259-272

[12] M. G. Ryskin, Diffractive $J / \psi$ Electroproduction in LLA QCD, Z. Phys. C57 (1993) 80-92

[13] S. J. Brodsky et al., Diffractive Leptoproduction of Vector Meson in QCD , Phys. Rev. D50 (1994) 3134-3180

[14] M. G. Ryskin, R. G. Roberts, A. D. Martin , E. M. Levin, Diffractive $J / \psi$ Photoproduction as a probe of the Gluon Density, European Phys. J. C76 (1997) 231-239

[15] A. D. Martin, M. G. Ryskin, T. Teubner, Upsilon Photoproduction at HERA Compared to Estimates of Perturbative QCD, Phys. Let. B454 (1999) 339-345 
[16] M. M. Block, K. Kang, A. R. White, The Current Status of High- Energy Elastic Scattering, Int. J. of Mod. Phys. A7 (1992) 44494476

[17] Erasmo Ferreira, Uri Maor, An Investigation of The Hard Contribution of $\varnothing$ Photoproduction, Phys. Let. B470 (1999) 200- 208

[18] D. ASton et al., Photoproduction of $k^{+} k^{-}$Pairs on Hydrogen at Photon Energies 20 to $36 \mathrm{GeV}$, Nucl. Phys. B172 (1980) 1-12

[19] M. Atkinson et al.(Omega Photon Coll.), The $\rho$ (1600) In The Reaction $\gamma p \rightarrow \pi^{0} \pi^{0} p$ At Photon Energies of 20-70 GeV, Z. Phys. C27 (1985) 233-238

[20] J. Busentiz et al., High energy Photoproduction of $\pi^{+} \pi^{-} \pi^{0}, k^{+} k^{-}$and $p p^{-}$States, Phys. Rev, D40 (1989) 1-21

[21] Khalid M. A. Hamad, $Q^{2}$ Dependence of Diffractive $\rho$ Meson Electroproduction, Eur. Phys. J. C72 (2012) 1927-1932

[22] A. Donnachie, G. Dosch, P. Landshoff, O. Naachtmann, Pomeron Phys. and QCD, Cambridge Unv. Press (2002)

[23] A. Donnachie, P. Landshoff, Small $\times$ Two Pomerons Phys. Lett. B437 (1998) 408-416

[24] I. Ivanov, N. Nikolaev, A. Savin, Diffractive Vector Meson Production at HERA from Soft to Hard QCD, Phys. Particles and Nuclei 37 (2005) $1-85$

[25] M. Block, E. Gregores, G. Pandari, PhotonProton and Photon - Photon scattering from Nucleon-Nucleon forward amplitude, Phys. Rev. D60 (1999) 54024-54037

[26] M. Gluck, E. Reya and A. Vogt, Dynamical distributions revisited Eur. Phys. J. C5 (1998) 461-470

[27] A. D. Martin, R. G. Roberts, W. J. Stirling and R. S. Thorne; Partons: a new analysis, Eur. Phys. J. C4 (1998) 463-496 


\title{
تكون الميزون المتجة فاي من تصادم الفوتون مع البروتون
}

\author{
خالا محمد عبد حمد، حسام محمد صداع حمد \\ قسم الفيزياء، كلية العلوم، جامعة الانبار، العر اق
}

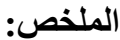

في هذا النموذج تم حساب المقطع العرضي لإنتاج الميزون فاي بواسطة الفوتون. حيث تم حساب المساهمة الناتجة من تبادل البومرون الناعم باستخدام نموذج DL. لقد وجد أن هذا النموذج يعطي نتائج تفوق النتائج التجريبية. لذا فقد نم استخدام معامل تصحيح يعتمد على كتل الميزونات م وض لتعديل نموذج DL. كما تم حساب مساهمة البومرون الصلب باستخدام نموذج pQCD. تم الحصول على نوافق معقول بين النموذج و النتائج العملية. 